SHAHRIAR AFANDIZADEH ZARGARI, Ph.D.

E-mail: zargari@iust.ac.ir

NAZLI DEHGHANI, Ph.D.

E-mail: ndehghani@iust.ac.ir

HAMID MIRZAHOSSEIN, Ph.D. Candidate

E-mail: mirzahossein@iust.ac.ir

Department of Transportation Planning and Engineering,

School of Civil Engineering

Iran University of Science and Technology Narmak, Tehran,

1684613114, Iran

MASOUD HAMEDI, Ph.D.

E-mail: masoud@umd.edu

Civil \& Environmental Engineering

University Of Maryland

1124D Glenn L. Martin Hall, College Park, MD, 20742, USA
Intelligent Transport Systems (ITS)

Preliminary Communication

Submitted: Jan. 5, 2015

Approved: Feb. 2, 2016

\title{
IMPROVING SCATS OPERATION DURING CONGESTION PERIODS USING INTERNAL/EXTERNAL TRAFFIC METERING STRATEGY
}

\begin{abstract}
Traffic metering is one of the effective strategies of preventing gridlock at urban signalized intersections during oversaturated conditions. This strategy could be implemented by adjusting signal timing schemes of the connected intersections of congested network in dynamic setting. This paper demonstrates the benefits of internal/external traffic metering strategy on a real case study in Tehran, the capital of Iran. For this purpose, the model outputs have been considered as input to set of SCATS scenarios for signal timing. In each test case the system is forced to use plans obtained from the model instead of using common built-in plans that had been used before, and the performance is measured using the VISSIM simulator to show differences. The results show significant improvement in network average travel time when using internal/external traffic metering strategy. Additionally, the average queue lengths are maintained near the optimal level since the model utilizes upstream arterial capacity.
\end{abstract}

\section{KEY WORDS}

traffic metering; gridlock; signal timing; SCATS subject classification; VISSIM;

\section{INTRODUCTION}

Limiting the inbound traffic flow is one of the travel demand management (TDM) approaches for preventing gridlock at intersections and their joint links. This approach can be applied in both isolated and integrated intersections. In dense networks with uniform congestion distribution, managing the entering traffic flow may well result in green time allocation that finally results in increased network performance.
There are many studies in the field of "traffic signal optimization" accomplished by researchers in the past two decades. In this regard, Gal-Tzur et al. offered a signal control method based on traffic metering. They presented a model to design signal timing for congested urban network in which one or more intersections may reach super-saturated condition compared to other intersections [1]. The main idea behind the approach was to restrict the inbound volume to the capacity of critical intersection in order to prevent the gridlock in urban transportation network. The metering process enables the system operators to detect the location and length of queues to adjust paths for creating appropriate relief buffers. In general, this strategy is named "queue location management". Traffic simulation and signal timing optimization programs like TRANSYT use the mathematical model designed and incorporated to implement the approach.

In the method developed by Chang and Lieberman [2], a non-linear programming model is hired to adjust the duration of arterial green period for each cycle. In this situation, the actual length of queues in each saturation approach always has a value very close to the optimal queue length that is calculated by mixed integer linear programming (MILP).

According to Daganzo theory [3] when network is in a congested state, even the smallest increase in the input volume beyond the critical value may have significant impact on the traffic condition and result in deterioration of the network performance. In such circumstances, where the system is unstable, controlling 
the input flow rate by hiring metering strategies on the boundary area is essential to keep the congestion manageable.

In this regard, the effectiveness of Daganzo theory has been presented in SCATS software [4] by Luke and Green. The purpose of this study was to evaluate the network-wide assignment strategies in an equilibrium state to reduce total delay. In a case study presented by Luke and Green, the methodology for metering and balancing traffic based on VR83 in SCATS software was investigated to reduce the delay in the network of Melbourne. SCATS VR83 employs DS (a measure of congestion behind the stop line showing the saturation degree) and a factor called Vo/Vk (representing the ratio of maximum output volume to the observed volume) to determine the time for adjusting signal scheduling scheme. VR83 re-distributes the green time when the intersection output faces limitations caused by congestion downstream. In this case, more green time may be given to the opposite approach that faces less congestion. One of the disadvantages of this method is the fact that the green time redistribution just depends on DS and the Vo/Vk ratio, where data are merely received from detectors behind the stop line. In order to avoid wasting green time and to improve the performance of the entire network, it is necessary to consider queue spillback and the optimal queue length in metering and also in flow control strategies.

Recently, Ekbatani[5] used the fundamental diagram of the urban networks for feedback-based gating control. In this study, gating was applied to avoid over-saturation in the urban network with the objective of maximizing the system output.

Also, He, Jiajia and Zaien Hou [6] in their studies, with one synthetic objective function containing the delay time, stops and network capacity, employ the ant colony method to solve the traffic signal timing optimization problem. Furthermore, Varia et al. [7] used the genetic algorithm for solving the optimization problem that solves traffic signal and dynamic flow simultaneously in urban congested network.

However, many publications in the area of signal control in networks, congestion management and queue spillback control can be found in literature. Nonetheless comprehensive modelling for joint optimization of congestion in the high priority sub-network (protected network) and upstream arteries (peripheral network) has not been considered. Moreover, optimal use of upstream capacity for flow buffering did not receive sufficient attention in previous studies.

Thus, in order to address the shortcomings of the current models, this paper proposes a solution including metering based on signal control models for urban networks that consider optimal conditions both upstream and downstream concurrently by monitoring the conditions of upstream inbound links continuously. In this situation, not only the queue spillback is prevented, but also their utilization is maximized due to queue length optimization. In other words, metering and limiting the inbound volume will help to keep the downstream network (also known as the protected network) far from the gridlock state and this will improve the overall system performance.

In this regard, this paper proposed a model that has been applied to factual network with an actual scenario in the city of Tehran based on real data. Figure 1 exhibits the proposed model components and the solution steps in a flowchart. As shown is Figure 1, in internal/external metering strategy the entire urban network is divided into two main parts referred to as protected and peripheral networks.

The main objective for the proposed model is to maintain the congestion of the protected network lower than the critical level by restricting inbound flow. However, traffic metering may cause queue spillback and disturb traffic conditions in the peripheral network, but the model applies queue optimization procedures to remedy the adverse metering impacts. Other components of the model demonstrated in Figure 1 include estimating congestion index in the protected network, queue length estimation in the peripheral network and solving the internal/external metering model for the entire network.

\section{INTERNAL/EXTERNAL TRAFFIC METERING MODEL IN URBAN NETWORK}

In this section the two main parts of the model are discussed. The formulation for the first part is as follows [2]:

$\operatorname{Max} \sum\left[\alpha_{i} \delta_{i}+\bar{\alpha}_{1} \bar{\delta}_{1}\right]$

Subject to:

$p_{0, i} \geq b_{11, i} \quad i=1, n-1$

$p_{0, i} \leq b_{7, i} \quad i=1, n-1$

$\delta_{i} \geq b_{0, i}-b_{1, i} p_{0, i}$ for saturated approaches

$\delta_{i} \leq b_{2, i}-b_{3, i} p_{0, i}$

$\delta_{i} \geq b_{4, i}-b_{5, i} p_{0, i}$ for under-saturated approaches (1-5)

$\delta_{i} \leq b_{0, i}-b_{1, i} p_{0, i}$

Poi $=$ rate of queue length $\mathrm{qi} / \mathrm{Li}$

Lieberman provides both upper and lower bounds for the above conditions [2] where:

$\delta_{i}$ : offset between two consecutive links in one direction

$\overline{\delta_{1}}$ : offset between two consecutive links in opposite direction

$\alpha_{i}, \overline{\alpha_{i}}:$ Related weights 


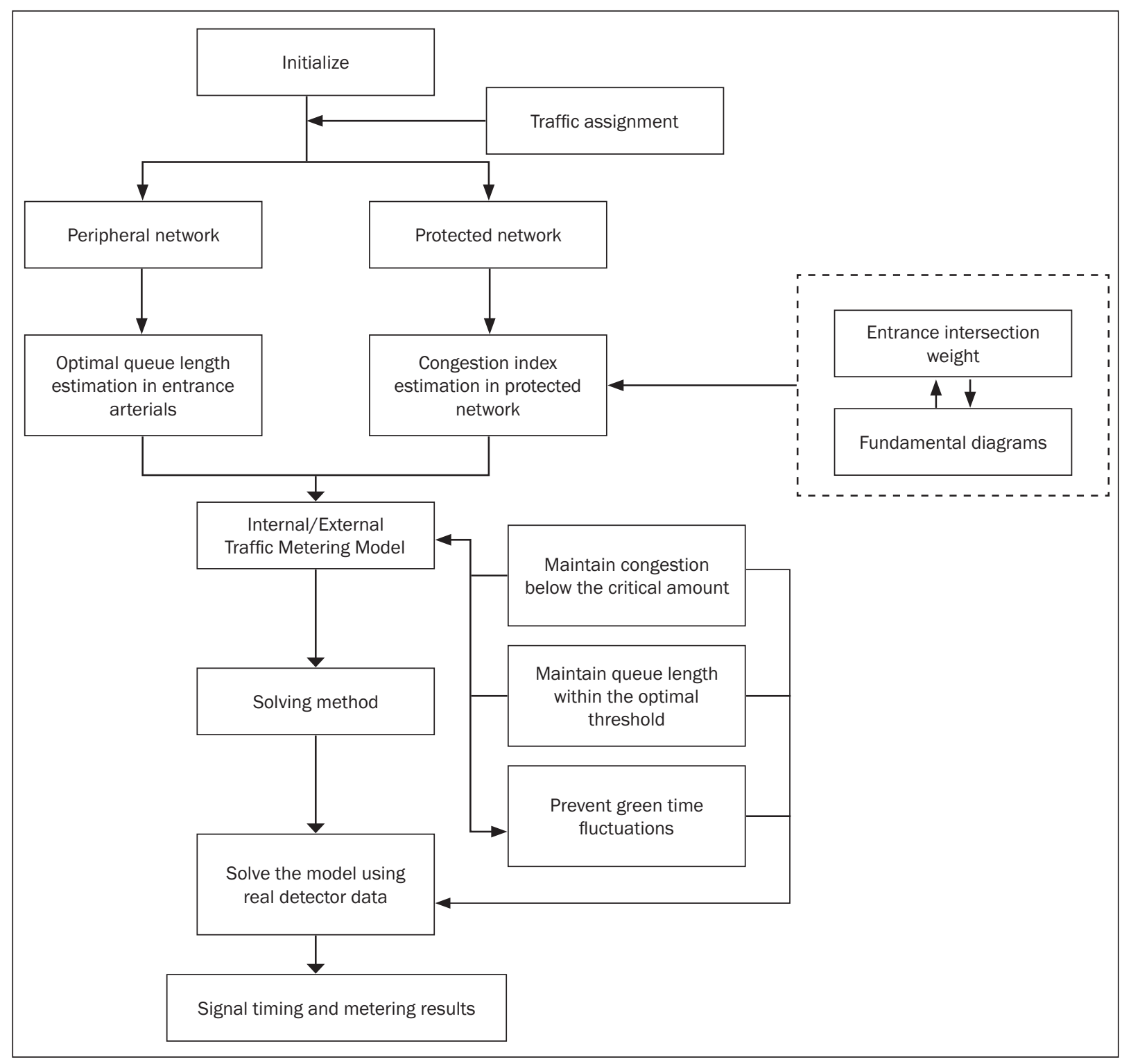

Figure 1 - Flowchart for solving the model in a real network

Objective function for the first part is to maximize the offset between two consecutive intersections. In the proposed model of this paper, the queue length is estimated in each cycle based on the Afandizadeh et al. research results [8] that were calibrated and applied for the intersections network of Tehran. The main advantage of the proposed model is queue spillback prevention in the upstream intersections by utilizing the capacity of the upstream links in the network. Most of the previous studies had ignored spillback of the flow to the surrounding areas of the protected network.

In the second part, the outputs of the first part including the optimal length of queues are considered as inputs to the second part which is as follows:

$$
\begin{aligned}
& \max \sum_{j=1}^{m} w_{j} q_{i n j} / T T S_{c r i}-w_{i} \sum_{i=1}^{n}\left(p_{0, i}-\hat{p}_{0, i}\right)^{2}- \\
& -\sum_{i=1}^{n}\left|\left(G_{B, i}-G_{B, i-1}\right) / C\right|
\end{aligned}
$$

Subject to:

$$
G_{B, i} \geq\left|G_{B, i}\right| \min
$$

$G_{B, i} \leq\left|G_{B, i}\right| \max$

$R_{A}=\frac{(L N)_{B}}{(L N)_{A}}$

$H_{B, 1}=R_{A} S^{(a)} \frac{S_{v}}{L}(1-P j)$

(2) $\quad H_{B, 3}=-\left(G_{B, i}-s\right) R_{A} S^{(a)} P_{B} \frac{S_{V}}{L}+\frac{\tau}{L N} \frac{S_{V}}{L}$
$H_{B, 2}=S^{(a)} \frac{S_{V}}{L}$ 


$$
\begin{aligned}
& \Delta p_{0}=H_{B, 1}\left(G_{B}-s\right)-H_{B, 2}\left(G_{A}-s\right)+H_{B, 3} \\
& H_{B, 4}=H_{B, 3}+s\left(H_{B, 2}-H_{B, 1}\right) \\
& \Delta p_{0}=H_{B, 1} G_{B}-H_{B, 2} G_{A}+H_{B, 4}
\end{aligned}
$$

where:

TTScri - congestion threshold in the protected network (veh.);

$\Delta p_{o, i}$ - difference in standing queue length in one cycle;

i $\quad$-index of saturated links;

$j \quad$-index of entrance arterials into the protected network;

wi - related weight;

$p_{o, i} \quad$ - standing queue length rate;

$\hat{p}_{o, i} \quad$ - optimal standing queue length rate;

$\mathrm{GBi} \quad$ - setting green time at intersection i(s);

wj - relative weight of intersection j from series of entrance intersections;

C - cycle time (sec);

$\mathrm{qin}_{\mathrm{j}} \quad$ - ratio of incoming volume from intersection $\mathrm{j}$ to the total incoming volume through the protected network (veh);

(LN)A - number of lanes at downstream intersection;

(LN)B - number of lanes at upstream intersection:

pj - turning vehicles from major street to $m$ nor streets at intersection $\mathrm{j}$ (percent)

P - percent of turning vehicles at upstream intersection.

The objective function of the second part of the model has three components. The first component accounts for congestion in the entire network based on controlling green time of entrance intersections under the critical congestion. The second and the third components determine optimal queue conditions in upstream arterial and prevent sudden fluctuations in consecutive green time. The last two components of the objective function are subject to conditions of arterial routes that are applied to the network for each input arterial link.

Furthermore, a new set of constraints have been added to allow for maximizing input flow to the network. The new constraints include the following equations.

$$
\begin{aligned}
& T T S \leq T_{\text {cri }} \\
& T T S=A \cdot N(t)+\varepsilon_{1} \\
& N(t)=\sum_{j=1}^{m} q_{i n j}+q_{d}-q_{\text {out }} \\
& \operatorname{qin}_{j}=L N_{j} \frac{G_{j}-S}{h} P_{j}+\tau \\
& \tau=\frac{C_{a}-G_{j}+S}{h} \cdot L N_{c 1} \cdot p_{c 1}+\frac{C_{a}-G_{j}+S}{h} \cdot L N_{c 2} \cdot p_{c 2}
\end{aligned}
$$

where:

$$
\begin{array}{ll}
T T S S_{c r i} & \text { - critical amount of TTS based on } \\
& \text { fundamental diagrams; } \\
A & \text { - error factor; } \\
\varepsilon_{1} & \text { - detectors error factor; } \\
\mathrm{j} & \text { - index of entrance intersection; } \\
\mathrm{LN}_{\mathrm{j}} & \text { - number of lanes in entrance arterials; } \\
\mathrm{m} & \text { - number of entrance intersections; } \\
\mathrm{G} & \text { - green time of entrance main approach (s); } \\
\mathrm{s} & \text { - lost time (s); } \\
\mathrm{h} & \text { - saturated headway (sec/veh); } \\
\mathrm{pj} & \text { - turning vehicles from major street to } \\
& \quad \text { minor streets at intersection j(percent); } \\
\mathrm{q}_{\mathrm{d}} & \text { - uncontrolled flows (veh/h); } \\
\mathrm{q}_{\text {out }} & \text { - output flow from protected } \\
& \text { network(veh/hr); } \\
\mathrm{w}_{\mathrm{j}} & \text { - related weight (entrance intersections); } \\
\mathrm{C} & \text { - cycle time (sec). }
\end{array}
$$

These new constraints will culminate so that congestion under the protected network can never be more than critical congestion. This issue is of vital importance, because if congestion occurs in the protective area, recovering of the system and switching back to the uncongested mode takes time. This will reduce the whole system productivity

\section{APPLICATION OF MODEL IN IMPROVING PERFORMANCE OF SCATS}

In order to study the proposed model in practice using SCATS software, a part of Tehran transportation network has been used. The location of case study is presented in Figure 2. This area is one of the best areas for determining the model results in Tehran, since most of the intersections are connected to SCATS. Schematic view of study area built in VISSIM is shown in Figure 2 where arrows show the arteries and spots show the intersections.

Different scenarios have been built and tested using VISSIM traffic simulator for implementing the pro-

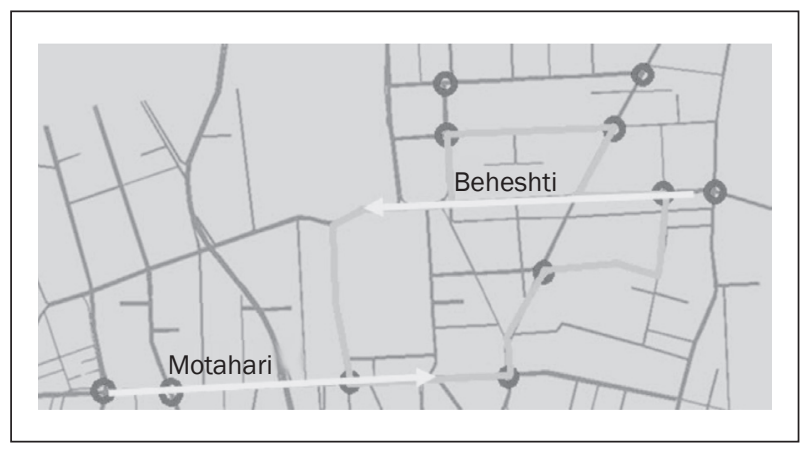

Figure 2 - Study area and the boundary of the protected network in Tehran 
posed model. The following information and sources are used in creating the test cases:

- Physical network data: nodes and links are created based on high-resolution satellite images of the area as well as AutoCAD maps. Additionally, in order to insure appropriate lane mapping and geometry alignment, the field visits were conducted and video surveillance footage was used.

- Travel demand data: the study area includes eleven intelligent intersections connected to SCATS, origin-destination (OD) data for the city of Tehran [9] were used and volumes for the desired links were extracted from the dataset.

- In a study conducted by Irannezhad and Moravveji [10], adjusted Origin-Destination data for Tehran were estimated based on the traffic volume statistics generated by the following resources:

- Traffic counters on eleven main highways of Tehran,

- Traffic volume data extracted from OCR cameras around the congestion pricing zone of Tehran,

- Traffic volume data around the restricted traffic zone of Tehran (surveillance in November 2011),

- Traffic counts in Hakim, Hemmat, Yadegar, Sheikhfazlolah and Chamran highways (surveillance in November 2011) [10].

According to the mentioned data, the adjusted origin-destination data were allocated to the entire network. A sub-matrix was extracted for the desired protected network using TRANSCAD software which was used as input into VISSIM. The dynamic assignment feature of VISSIM that uses virtual origins and destinations (parking spaces) was applied to generate link volumes. After the assignment process, transit and traffic volumes and turning moves in each entrance were calculated for the arteries.

\subsection{Applying the model}

The solution approach was coded in MATLAB and applied to the test network with five input arterials. The MATLAB code interacts with VISSIM through a file interface to measure the overall performance for one cycle. At each stage VISSIM provides the following measures to the MATLAB code:

- Occupancy at stop line detectors;

- Occupancy at queue detectors;

- Phase change time at the main intersection;

- Phase change time at the upstream intersection;

- Detection pulse at stop line detector;

- Detection pulse at queue detector.

Each iteration of the program includes determining the arterial inputs, retrieving traffic volume data, green time calculation and traffic state updating. The outputs of these steps are the ratio of the optimal queue

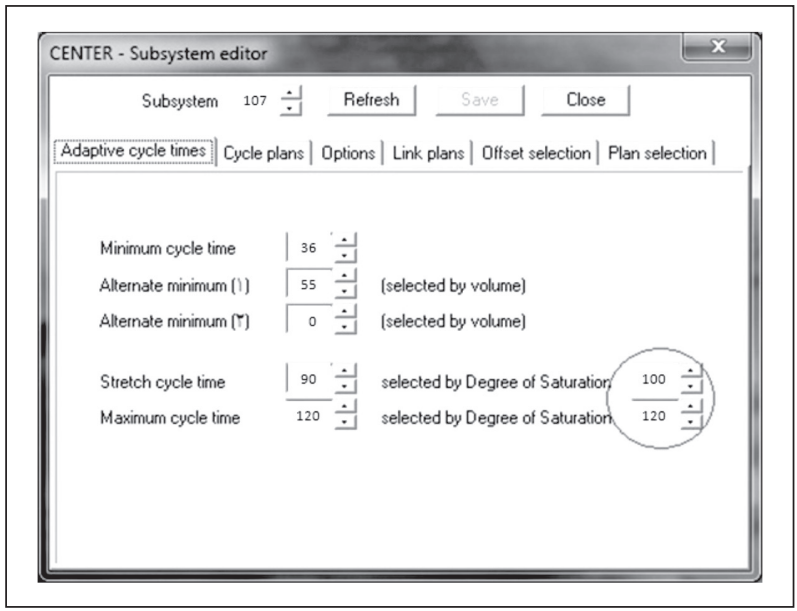

Figure 3 - Selection of DS1 and DS2

length and the optimal offset in each single entrance of arteries.

In this phase, the length of queue for each inbound link is estimated based on the coded solution for the intended network. Also, to satisfy the optimality conditions, the estimated value for the queue length must be in the proximity of the value that was calculated during the first phase of the solution.

\subsection{The model implementation in SCATS}

In order to implement the internal/external metering model in the SCATS system, the concept of creating congestion in SCATS has been applied. To detect the congestion conditions in the SCATS system a feature called variation routine (VR) has been used.

This tool allows making changes to the signal timing in response to desired conditions which may override the predetermined plans. Such scenarios are entered in the system as conditional logical statements in "if / then" format. The system will take action if the criteria for a condition are satisfied and will withdraw the change upon removal of the condition. By using this tool several parameters such as cycle length and saturation level may be included in creating the conditional scenarios. Therefore, when congestion is detected at an intersection, the central intelligent control system of SCATS requested to take one of the following actions to adjust the signal timing and increase throughput:

In SCATS system, the maximum cycle length during traffic congestion periods depends on the maximum degree of saturation (DS) calculated by the system. Consequently, two values for DS are determined by investigating the traffic conditions of intersection during the peak traffic. Figure 3 is a screenshot showing values of DS1 and DS2 in the Subsystem editor section.

The values available in the corresponding action can be read and implemented by Variation 67 , which is DS value for the target approach. In the defined sys- 
tem, if the calculated DS is equal to the defined value, the corresponding action shall be taken by setting "below cycle time". Variation 74 can also be used to define actions based on congestion index for one or all detectors available in an approach to execute the desired plan.

It should be noted that SCATS allows defining a split plan for partitioning phases based on expert opinion. However, this paper uses the results of solving the model for calculating the average of signal timing schedules based on congestion conditions as input parameters of the split plan. Thus, upon detecting congestion at downstream intersections, VR74 is used to override the plans and start metering the upstream traffic flow to prevent gridlock.

\subsection{Discussion}

In order to evaluate the network performance when the internal/external traffic metering strategy is applied and its comparison with the previous case (without internal/external traffic metering strategy), two parameters of the input arteries have been estimated. These two are the average travel time and the average queue length.

For this purpose, the travel time of two arteries Beheshti and Motahari that have been shown with arrows in Figure 1 are considered. The travel time as the main component of the network have been measured by probe vehicle in the morning peak hours.

Also, after applying internal/external traffic metering strategy the same travel time measurement was repeated at peak hour and on the same day of the week. Figures 3 and 4 show the results of travel time comparison before and after applying the internal/ external traffic metering strategy (IETMS) in the mentioned arteries.

As the results show, the travel times of the two primary arteries constituting internal network have been improved by $15.4 \%$ and $6.3 \%$ after applying the proposed approach.

Moreover, in the proposed model, the focus of queue length optimization is based on the inbound links and they have been calculated based on image

Table 1 - Comparison of queue length on entrance arteries

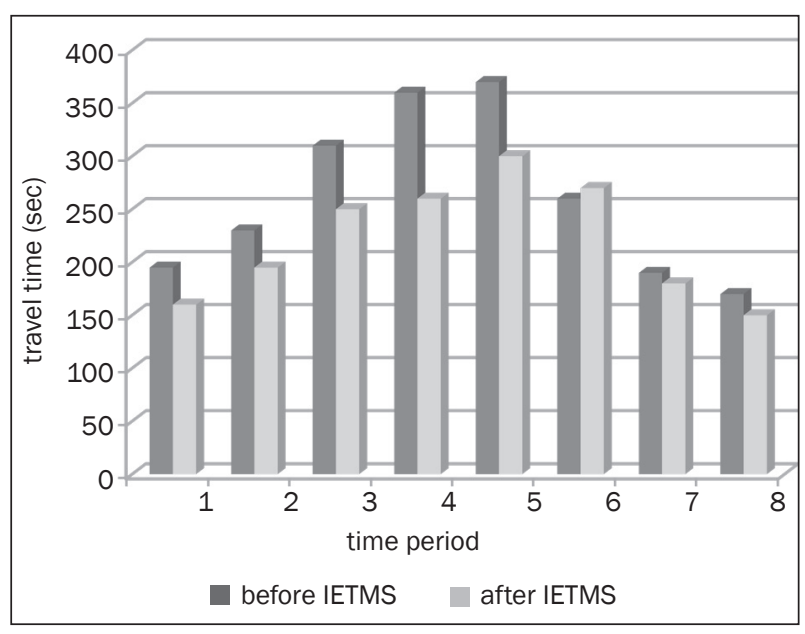

Figure 4 - Average Travel Time in Beheshti Avenue

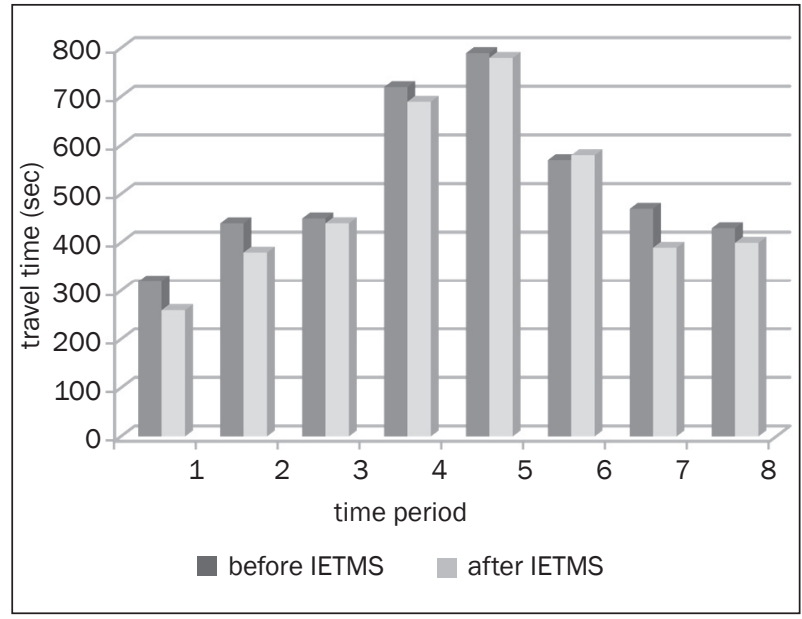

Figure 5 - Average Travel Time in Motahari Avenue

data received from traffic surveillance cameras. Thus, during peak hours and based on cameras data, the queue length is measured at certain regular intervals and also compared with the predicted queue length of the model. Similar comparison is repeated when internal/external traffic metering strategy is applied to the same time period.

\begin{tabular}{|c|c|c|c|c|c||}
\hline Arterial name & $(1)$ & $(2)$ & $(3)$ & $(4)$ & $(5)$ \\
\hline \hline & $\begin{array}{c}\text { Optimal queue } \\
\text { length-meter } \\
\text { (No. of vehicles) }\end{array}$ & $\begin{array}{c}\text { Avg. queue length } \\
\text { before } \\
\text { IETMS-meter } \\
\text { (No. of vehicles) }\end{array}$ & $\begin{array}{c}\text { Avg. queue length } \\
\text { after IETMS-meter } \\
\text { (No. of vehicles) }\end{array}$ & $\begin{array}{c}\text { Difference } \\
\text { between column } \\
\text { numbers } \\
\text { (1) - (2) } \\
\text { (No. of vehicles) }\end{array}$ & $\begin{array}{c}\text { Difference } \\
\text { between column } \\
\text { numbers } \\
\text { (1) - (3). of vehicles) } \\
\text { (No. on }\end{array}$ \\
\hline Beheshti & 35 & 30 & 36 & 5 & 1 \\
\hline Motahari & 49 & 43 & 53 & 6 & 4 \\
\hline Sohrevardi & 58 & 50 & 63 & 8 & 5 \\
\hline Saboonchi & 26 & 21 & 30 & 5 & 4 \\
\hline
\end{tabular}


The results are summarized in Table 1. It shows that after applying internal/external traffic metering strategy, the difference between average queue length and its optimal rates has decreased.

As shown in Table 1, for instance, the difference between the optimal queue length meter based on the number of vehicles assigned to column (1) and the average queue length before IETMS meter assigned to column (2) is 5 for Beheshti Street that is shown in column (4). Also, the difference between the optimal queue length meter based on the number of vehicles assigned to column (1) and the average queue length after IETMS meter implementation assigned to column (2) is 1 for Beheshti Avenue that is shown in column (5). These calculations could be used as capacity indication of the inbound arteries that show better operation after applying the introduced metering methodology.

\section{CONCLUSION}

This paper shows the application of internal/external traffic metering strategy model in the real urban transportation network. Also, the network gridlock is prevented by limiting the inbound flow beyond target levels and by implementing the metering models.

Moreover, the upstream queue levels in the input links are maintained within optimal limits by adjusting the signal timing to avoid queue spillback which is critical in dense urban transportation network with capacity constraints.

In addition, this model used a real case study in Tehran and demonstrated that network performance indicators such as the average travel time and the average length of the queue can be improved. Also, it shows that even in the presence of commercial Intelligent Transportation Systems (ITS) the software for signal timing management like SCATS, internal/external traffic metering may improve the network performance.
Finally, it would certainly be desirable to be able to consider online implementation of this model in a network as future research, where all the intersections are equipped with queue detectors and connected to SCATS.

\section{REFERENCES}

[1] Gal-Tzur A, Mahalel D, Prashker JN. Signal design for congested networks based on metering; 1993.

[2] Lieberman E, Chang J, Prassas E. Formulation of real-time control policy for oversaturated arterials. Transportation Research Record: Journal of the Transportation Research Board. 2000;(1727):77-88.

[3] Daganzo CF. Urban gridlock: macroscopic modeling and mitigation approaches. Transportation Research Part B: Methodological. 2007;41(1):49-62.

[4] Luk J, Green D. Balancing traffic density in a signalised network; 2010.

[5] Keyvan-Ekbatani M, Kouvelas A, Papamichail I, Papageorgiou M. Exploiting the fundamental diagram of urban networks for feedback-based gating. Transportation Research Part B: Methodological. 2012;46(10):1393-403.

[6] He J, Hou Z. Ant colony algorithm for traffic signal timing optimization. Advances in Engineering Software. 2012;43(1):14-8.

[7] Varia H, Gundaliya P, Dhingra S. Application of genetic algorithms for joint optimization of signal setting parameters and dynamic traffic assignment for the real network data. Research in Transportation Economics. 2013;38(1):35-44.

[8] Afandizadeh S, Latifi M, Dehghani N. Real time queue estimation model in saturated urban arterials in Tehran. Journal of transportation research. 2013;4(1):365.

[9] TCTTS TCTaTSC-. Link Performance Function Studies; 2014.

[10] Irannezhad E, Mousavi SH, Moraveji G, editors. Updating OD Matrix based on traffic counts for evaluating traffic management plans in Tehran. Proceedings of 11th International Traffic \& Transportation Conference, Tehran, Iran; 2012 